Mathematical Modelling and Analysis

Volume 5, 2000, PAGes 153-163

(C) 2000 Technika

\title{
ON WELL-POSEDNESS OF NONLINEAR CONJUGATION BOUNDARY VALUE PROBLEM FOR ANALYTIC FUNCTIONS
}

\author{
S.V. ROGOSIN \\ Department of Mathematics and Mechanics, Belarusian State University
}

Fr. Skaryny ave 4, 220050, Minsk, Belarus

E-mail: rogosin@mmf.bsu.unibel.by

Received October 19, 1999

\begin{abstract}
We consider power type nonlinear conjugation problem for analytic functions. Our main question is to make this problem well-posed, i.e. to find such classes of functions in which this problem possesses a unique solution. These classes are those with prescribed collections of zeros in the domains and/or on their boundaries.
\end{abstract}

\section{INTRODUCTION}

Linear boundary value problems for analytic functions seems to be now classical subject of Analysis (for its advanced description see e.g. [1] - [2]). The study of corresponding nonlinear problems was stimulated by applications in different questions of elasticity, elasto-plasticity, hydrodynamics, composite materials etc. (we refer the reader in to surveys [3], [4], [5], to books [6], [7], and to the extensive bibliography therein). Among the approaches presented in this area are those of constructive nature (see e.g. [3], [4], [7]), in which are generalized the analytic methods applied for the linear case. It was appeared that such generalizations are not always applicable even to most simple nonlinear problems. Some new features occur which need a new technique. Therefore the theory of nonlinear boundary value problems is far from completeness.

One of the important questions at the study of nonlinear boundary value problems is to find proper classes for solutions. It was shown (see e.g. [4], [8]) that the solvability of these problems highly depends on the distribution 
of zeros of the solutions.

Here we study this dependence in the case of power type nonlinear conjugation problem (the corresponding linear problem is known also as Riemann problem, or Hilbert problem). It was investigated by many authors (see e.g. [9], [10], [11], [7]), but our approach slightly differs from those used in the above cited papers.

We describe zero distribution in the domains and/or on their boundaries in the cases when the explicit solution can be constructed. On this base we discover the functional classes in which the problem under discussion is well-posed.

\section{NONLINEAR CONJUGATION PROBLEM.}

We consider here the following problem: let $L$ be a closed simple smooth curve divided the complex plane $\mathbb{C}$ onto two domains $D^{+} \ni 0, D^{-} \ni \infty$; given two real numbers $\alpha, \beta \in \mathbb{R} \backslash\{0\}$, and two Hölder-continuous functions $G, g$ find functions $\Phi^{+}, \Phi^{-}$, analytic in $D^{+}, D^{-}$respectively satisfying the boundary condition

$$
\left[\Phi^{+}(t)\right]^{\alpha}=G(t)\left[\Phi^{-}(t)\right]^{\beta}+g(t), t \in L,
$$

It should be noted that if $\alpha, \beta$ have different signs, i.e. $\alpha \beta<0$, then (2.1) becomes the problem of another type. Besides, the case $\alpha, \beta \in \mathbb{R}_{-}$can be clearly reduced to the case of positive exponents. Therefore, we suppose in what follows that $\alpha, \beta \in \mathbb{R}_{+}$.

Following [4], [7] we say, that a pair of functions $\Phi^{+}, \Phi^{-}$belongs to the class $\mathcal{A}^{k, l}$, if these functions are analytic in $D^{+}, D^{-}$respectively, continuous up to the boundary, and have exactly $k, l$ zeros, which are situated into domains $D^{+}, D^{-}$respectively. If we additionally suppose that the functions of $\mathcal{A}^{k, l}$ are possible to be vanishing on the boundary (not necessarily of integer order), that we denote corresponding class by $\widetilde{\mathcal{A}}^{k, l}$.

Our idea is to find ALL POSSIBLE values of parameters $\alpha, \beta$, for which the problem (2.1) has a solution, rather than to prescribe the classes of solutions beforehand.

Let us assume that $z_{j}^{+} \in D^{+}, j=1, \ldots, k\left(z_{j}^{-} \in D^{-}, j=1, \ldots, l_{1}\right.$, respectively) are zeros of $\Phi^{+}\left(\Phi^{-}\right)$, and $\Phi^{-}$has zero of order $l_{0}$ at infinity; $l=l_{0}+l_{1}$. By changing of unknown functions

$$
\left\{\begin{array}{l}
\Psi^{+}(z):=\left[\prod_{j=1}^{k}\left(z-z_{j}^{+}\right)\right]^{-1} \Phi^{+}(z), z \in D^{+}, \\
\Psi^{-}(z):=z^{l_{0}}\left[\prod_{j=1}^{l_{1}}\left(1-\frac{z_{j}^{-}}{z}\right)\right]^{-1} \Phi^{-}(z), z \in D^{-}
\end{array}\right.
$$


one can reduce (2.1) to the following boundary condition

$$
\left[\Psi^{+}(t)\right]^{\alpha}=\frac{\left[\prod_{j=1}^{l_{1}}\left(t-z_{j}^{-}\right)\right]^{\beta}}{\left[\prod_{j=1}^{k}\left(1-\frac{z_{j}^{+}}{t}\right)\right]^{\alpha}} G(t) t^{-k \alpha-l \beta}\left[\Psi^{-}(t)\right]^{\beta}+\frac{g(t)}{\left[\prod_{j=1}^{k}\left(t-z_{j}^{+}\right)\right]^{\alpha}}, t \in L,
$$

where the products $\left[\prod_{j=1}^{l_{1}} \ldots\right]^{\beta}\left(\left[\prod_{j=1}^{k} \cdots\right]^{\alpha}\right)$ in the first term in the right-hand

side are boundary values of arbitrary fixed in $D^{+}\left(D^{-}\right.$resp.) branches of corresponding multi-valued functions. The problem (2.3) has to be considered in the classes $\mathcal{A}^{k, l}$ or $\widetilde{\mathcal{A}}^{k, l}$ of functions having no zero in the domains. Therefore the following functions

$$
\left\{\begin{array}{l}
\Omega^{+}(z):=\left[\prod_{j=1}^{l_{1}}\left(z-z_{j}^{-}\right)\right]^{-\beta}\left[\Psi^{+}(z)\right]^{\alpha}, z \in D^{+} \\
\Omega^{-}(z):=\left[\prod_{j=1}^{k}\left(1-\frac{z_{j}^{+}}{z}\right)\right]^{-\alpha}\left[\Psi^{-}(z)\right]^{\beta}, z \in D^{-}
\end{array}\right.
$$

are single-valued in the corresponding domains. Thus the problem (2.3) is reduced to the equivalent linear boundary value problem

$$
\Omega^{+}(t)=G(t) t^{-k \alpha-l \beta} \Omega^{-}(t)+\frac{g(t)}{\left[\prod_{j=1}^{k}\left(t-z_{j}^{+}\right)\right]^{\alpha}\left[\prod_{j=1}^{l_{1}}\left(t-z_{j}^{-}\right)\right]^{\beta}}, t \in L,
$$

Denoting by $\chi:=\operatorname{wind}_{L} G$ the Cauchy index of the coefficient of (2.1), one gets three essentially different situations.

$1^{0}$. Let $k, l \in \mathbb{N}_{0}:=\{0,1, \ldots\}$ be such that

$$
\chi=k \alpha+l \beta .
$$

Then for any pair of such $(k, l)$ the index of the linear problem $(2.5)$ is equal to zero, hence (cf. e.g. [1, p.93]) this problem has the following analytic solution:

$$
\Omega^{ \pm}(z)=\exp \left\{\mathbf{S}^{ \pm}\left(\log G_{1}\right)(z)\left[\left(\mathbf{S}^{ \pm} g_{1}\right)(z)-C_{0}\right], z \in D^{ \pm},\right.
$$

where $\mathbf{S}^{ \pm}$is the Cauchy type operator for $z \in D^{ \pm}$respectively, $C_{0}$ is an arbitrary complex constant,

$$
G_{1}(t):=G(t) t^{-k \alpha-l \beta} ; g_{1}(t):=g(t)\left[\prod_{j=1}^{k}\left(t-z_{j}^{+}\right)\right]^{-\alpha}\left[\prod_{j=1}^{l_{1}}\left(t-z_{j}^{-}\right)\right]^{-\beta} .
$$


In order to have the solution of (2.7) belonging to $\mathcal{A}^{k, l}$ or $\widetilde{\mathcal{A}}^{k, l}$ one should choose the constant $C_{0}$ in accordance with one of the following "branching" condition (cf. [11]):

$$
\begin{aligned}
& C_{0} \notin\left(\mathbf{S}^{+} g_{1}\right)\left(\overline{D^{+}}\right) \cup\left(\mathbf{S}^{-} g_{1}\right)\left(\overline{D^{-}}\right), \\
& C_{0} \notin\left(\mathbf{S}^{+} g_{1}\right)\left(D^{+}\right) \cup\left(\mathbf{S}^{-} g_{1}\right)\left(D^{-}\right) .
\end{aligned}
$$

Since the sets in the right-hand sides of (2.8) and (2.9) are bounded both choices are possible.

Proposition 2.1. Let $(k, l) \in \mathbb{N}_{0} \times \mathbb{N}_{0}$ be such that the condition (2.6) does satisfy. Then the problem (2.1) has a solution

$$
\left\{\begin{array}{l}
\Phi^{+}(z)=\prod_{j=1}^{k}\left(z-z_{j}^{+}\right) \cdot\left[\prod_{j=1}^{l_{1}}\left(z-z_{j}^{-}\right)\right]^{\frac{\beta}{\alpha}}\left[\Omega^{+}(z)\right]^{\frac{1}{\alpha}}, z \in D^{+}, \\
\Phi^{-}(z)=z^{l_{0}} \prod_{j=1}^{l_{1}}\left(1-\frac{z_{j}^{-}}{z}\right) \cdot\left[\prod_{j=1}^{k}\left(1-\frac{z_{j}^{+}}{z}\right)\right]^{\frac{\alpha}{\beta}}\left[\Omega^{-}(z)\right]^{\frac{1}{\beta}}, z \in D^{-},
\end{array}\right.
$$

in the class $\mathcal{A}^{k, l}\left(\widetilde{\mathcal{A}}^{k, l}\right)$ if the complex constant $C_{0}$ is chosen as in (2.8) (as in (2.9) respectively). The functions $\left[\Omega^{+}\right]^{\frac{1}{\alpha}}$, and $\left[\Omega^{-}\right]^{\frac{1}{\beta}}$ are then any branches of the multi-valued functions with $\Omega^{ \pm}$delivered in (8).

$2^{0}$. Let $k, l \in \mathbb{N}_{0}$ be such that

$$
\chi_{0}:=\chi-k \alpha-l \beta \in \mathbb{Z} \backslash\{0\} .
$$

Then the coefficient of the problem (2.5) can be rewritten in the form

$$
G_{1}(t):=G(t) t^{-k \alpha-l \beta}=G_{0}(t) t^{\chi_{0}},
$$

where wind $_{0}=0$. Therefore one needs to solve the following auxiliary factorization problem:

$$
X^{+}(t)=t^{\chi_{0}} X^{-}(t), t \in L
$$

in the class $\widetilde{\mathcal{A}}^{0,0}$. It is clear that by assumption there is no solution of (2.11) in $\mathcal{A}^{0,0}$.

a) $\chi_{0} \in \mathbb{N}$.

It can be noted that we should suppose either $\Omega^{+}$having zeros on $L$, or $\Omega^{-}$ having them. Both are vanishing only at the points at which $g(t)=0$. 
Let us choose some points (for the sake of determines, different ones) $t_{1}, \ldots, t_{\chi_{0}} \in L$, at which

$$
\Omega^{+}\left(t_{j}\right) \cdot \Omega^{-}\left(t_{j}\right)=0, j=1, \ldots, \chi_{0} .
$$

Then we have the following partial solution of (2.11):

$$
\left\{\begin{array}{l}
X^{+}(z)=\prod_{j=1}^{\chi_{0}}\left(z-t_{j}\right), z \in D^{+} \\
X^{-}(z)=\prod_{j=1}^{\chi_{0}}\left(1-\frac{t_{j}}{z}\right), z \in D^{-}
\end{array}\right.
$$

By using (2.12) one can rewrite the boundary condition (2.5) as follows

$$
\begin{aligned}
\frac{\Omega^{+}(t)}{X^{+}(t) \exp \left\{\mathbf{S}^{+}\left(\log G_{0}\right)(t)\right\}} & -\frac{\Omega^{-}(t)}{X^{-}(t) \exp \left\{\mathbf{S}^{-}\left(\log G_{0}\right)(t)\right\}} \\
& =\frac{\tilde{\tilde{S}}(t)}{X^{+}(t) \exp \left\{\mathbf{S}^{+}\left(\log G_{0}\right)(t)\right\}}
\end{aligned}
$$

It is clear that the right-hand side of (2.13) is not in general integrable. To overcome this difficulty we interpolate $g_{2}(t):=g_{1}(t) \exp \left\{-\mathbf{S}^{+}\left(\log G_{0}\right)(t)\right\}$ by using of Lagrange polynomials:

$$
\frac{g_{2}(t)}{X^{+}(t)}:=\frac{g_{2}(t)}{\omega(t)}=\frac{\widehat{g(t)}}{\omega(t)}+\sum_{j=1}^{\chi_{0}} \frac{g_{2}\left(t_{j}\right)}{\omega^{\prime}\left(t_{j}\right)\left(t-t_{j}\right)},
$$

where $\widehat{g(t)}:=g_{2}(t)-\sum_{j=1}^{\chi_{0}} \frac{\omega(t) g_{2}\left(t_{j}\right)}{\omega^{\prime}\left(t_{j}\right)\left(t-t_{j}\right)}$. Applying then Plemelj-Sokhotsky formulas to $\widehat{g} \omega^{-1}$, and analytically continuing the equality (2.13) one is led to the following general solution of the problem (2.5):

$$
\left\{\begin{array}{l}
\Omega^{+}(z)=X^{+}(z) \exp \left\{\mathbf{S}^{+}\left(\log G_{0}\right)(z)\right\}\left[\widetilde{\mathbf{S}}^{+}(\widehat{g})(z)-C_{0}\right] \\
\Omega^{-}(z)=X^{-}(z) \exp \left\{\mathbf{S}^{-}\left(\log G_{0}\right)(z)\right\}\left[\widetilde{\mathbf{S}}^{-}(\widehat{g})(z)-C_{0}\right]
\end{array}\right.
$$

where

$$
\left\{\begin{array}{l}
\widetilde{\mathbf{S}}^{+}(\widehat{g})(z):=\mathbf{S}^{+}\left(\frac{\widehat{\underline{g}}}{\omega}\right)(z)-\sum_{j=1}^{\chi_{0}} \frac{g_{2}\left(t_{j}\right)+C_{j}}{\omega^{\prime}\left(t_{j}\right)\left(z-t_{j}\right)} \\
\widetilde{\mathbf{S}}^{-}(\widehat{g})(z):=\mathbf{S}^{+}\left(\frac{\widehat{\underline{g}}}{\omega}\right)(z)-\sum_{j=1}^{\chi_{0}} \frac{C_{j}}{\omega^{\prime}\left(t_{j}\right)\left(z-t_{j}\right)}
\end{array}\right.
$$

and the constants $C_{j}$ are chosen in the following way:

$$
\begin{array}{ll}
C_{j}=-g_{1}(t j), & \text { if we prescribe } \Omega^{+} \text {to be vanishing at } t_{j} \\
C_{j}=0, & \text { if we prescribe } \Omega^{-} \text {to be vanishing at } t_{j} .
\end{array}
$$


We suppose also that $C_{0}$ is taken as

$$
C_{0} \notin \widetilde{\mathbf{S}}^{+}\left(g_{2}\right)\left(\overline{D^{+}}\right) \cup \widetilde{\mathbf{S}}^{-}\left(g_{2}\right)\left(\overline{D^{-}}\right) .
$$

It should be noted that the branch of powers of the function $X^{+}$(of $X^{-}$ respectively) is an arbitrary taken in the complex plane with cuts connecting $t_{j}$ and $\infty$ (connecting $t_{j}$ and 0 resp.) and having no other common points with $L$ besides of $t_{j}$. The branches of the others functions are chosen as before.

b) $\chi_{0} \in-\mathbb{N}$.

It is not hard to see that the factorization problem (2.11) has no solution in $\widetilde{\mathcal{A}}^{0,0}$. But if one try to use "meromorphic on the boundary" solution of (2.11):

$$
\left\{\begin{array}{l}
X^{+}(z):=\left[\prod_{j=1}^{-\chi_{0}}\left(z-t_{j}\right)\right]^{-1}, z \in D^{+}, \\
X^{+}(z):=\left[\prod_{j=1}^{-\chi_{0}}\left(1-\frac{t_{j}}{z}\right)\right]^{-1}, z \in D^{-}
\end{array}\right.
$$

then it leads to very strong condition that the singular integral of the righthand side of (2.13) should have zero of at least first order at all $t_{j}$ th. To avoid this we modify our scheme. Let us choose a rational number $r$ such that

$$
0<r \leq \min \left\{\lambda_{G}, \lambda_{g}, 1-\epsilon\right\} ; r \cdot n=-\chi_{0},
$$

where $\epsilon \in(0,1)$ is any "sufficiently small" number, $n$ is a positive integer; $\lambda_{G}, \lambda_{g} \in(0,1]$ are Hölder exponents of the functions $G$ and $g$ respectively. Having chosen points $t_{1}, \ldots, t_{n} \in L$ we determine a solution of the factorization problem (2.11) in the form:

$$
\left\{\begin{aligned}
X^{+}(z) & :=\prod_{j=1}^{n}\left(z-t_{j}\right)^{-r}, z \in D^{+}, \\
X^{+}(z) & :=\prod_{j=1}^{n}\left(1-\frac{t_{j}}{z}\right)^{-r}, z \in D^{-} .
\end{aligned}\right.
$$

The branches of $\left(z-t_{j}\right)^{-r}$, and of $\left(1-\frac{t_{j}}{z}\right)^{-r}$ can be chosen in $\mathbb{C} \backslash\left(t_{j}, \infty\right)$, in $\mathbb{C} \backslash\left(0, t_{j}\right)$ respectively in such a way that

$$
\left(t-t_{j}\right)^{-r}=t^{-r}\left(1-\frac{t_{j}}{t}\right)^{-r}, \prod_{j=1}^{n} t^{-r}=t^{\chi_{0}} \text { for all } t \in L \backslash\left\{t_{1}, \ldots, t_{n}\right\} .
$$

Using the functions $X^{ \pm}$from (2.19) one gets immediately the following solution of the problem $(2.5)$ of the class $\widetilde{\mathcal{A}}^{0,0}$ :

$$
\left\{\begin{array}{l}
\Omega^{+}(z)=X^{+}(z) \exp \left\{\mathbf{S}^{+}\left(\log G_{0}\right)(z)\right\}\left[\mathbf{S}^{+}\left(g_{3}\right)(z)-C_{0}\right], z \in D^{+}, \\
\Omega^{-}(z)=X^{-}(z) \exp \left\{\mathbf{S}^{-}\left(\log G_{0}\right)(z)\right\}\left[\mathbf{S}^{-}\left(g_{3}\right)(z)-C_{0}\right], z \in D^{-},
\end{array}\right.
$$


with $g_{3}:=g_{1}\left(X^{+}\right)^{-1} \exp \left\{-\mathbf{S}^{+}\left(\log G_{0}\right)\right\}$. The constant $C_{0}$ have to be chosen as

$$
C_{0} \notin \mathbf{S}^{ \pm}\left(g_{3}\right)\left(D^{ \pm} \backslash\left\{t_{1}, \ldots, t_{n}\right\}\right) .
$$

Besides, the terms in square brackets in (2.21) should vanish at the points $t_{j}$. Together with Plemelj-Sokhotsky formulas and with (2.22) it leads to the following necessary and sufficient solvability conditions:

$$
C_{0}=\mathbf{S}\left(g_{3}\right)\left(t_{1}\right)=\cdots=\mathbf{S}\left(g_{3}\right)\left(t_{n}\right) \notin \mathbf{S}^{ \pm}\left(g_{3}\right)\left(D^{ \pm} \backslash\left\{t_{1}, \ldots, t_{n}\right\}\right),
$$

where $\mathbf{S}$ is a singular integral operator with Cauchy kernel.

Proposition 2.2. Let $(k, l) \in \mathbb{N}_{0} \times \mathbb{N}_{0}$ be such that $\chi_{0} \neq 0$ is an integer number.

If $\chi_{0} \in \mathbb{N}$ then the unique (up to the constant $C_{0}$ satisfied (2.17)) solution of the problem (2.1) of the class $\widetilde{\mathcal{A}}^{k, l}$ has the form (2.10) with $\Omega^{ \pm}$determined in (2.14).

If $\chi_{0} \in-\mathbb{N}$ then then the solution of the problem (2.1) can be also delivered by the formula (2.10) with $\Omega^{ \pm}$given in (2.21). This solution belongs to $\widetilde{\mathcal{A}}^{k, l}$ if the solvability conditions (2.23) do satisfy.

Remark 2.1. The proposed scheme leads us to the solution with "maximally" possible order of zeros at certain points on the boundary, namely

$$
r_{+}:=\frac{\min \left\{\lambda_{G}, \lambda_{g}\right\}}{\alpha}, r_{-}:=\frac{\min \left\{\lambda_{G}, \lambda_{g}\right\}}{\beta},
$$

for $\Phi^{+}, \Phi^{-}$respectively. It means that from general conditions for $G, g$ it can be deduced only such order of zeros for solution. If $G, g$ have smoother behaviour at certain points then the solution can have there zeros of bigger order.

$3^{0}$. Let $(k, l) \in \mathbb{N}_{0} \times \mathbb{N}_{0}$ be such that

$$
\chi_{0} \in \mathbb{R} \backslash \mathbb{Z}
$$

We are led again to the factorization problem (2.11), but with noninteger exponent $\chi_{0}$.

a) $\chi_{0} \in \mathbb{R}_{+} \backslash \mathbb{Z}$, i.e. $\chi_{0}$ is noninteger positive number. There are in principle two approaches to solve the factorization problem (2.11).

i) Let us choose $n=\left[\chi_{0}\right]$ points $t_{j}$ on $L$, and a point $t_{0} \in L \backslash\left\{t_{1}, \ldots, t_{n}\right\}$ such that

$$
\left\{\begin{array}{l}
X^{+}(z):=\left(z-t_{0}\right)^{\chi_{0}-\left[\chi_{0}\right]} \prod_{j=1}^{n}\left(z-t_{j}\right), z \in D^{+}, \\
X^{+}(z):=\left(1-\frac{t_{0}}{z}\right)^{\chi_{0}-\left[\chi_{0}\right]} \prod_{j=1}^{n}\left(1-\frac{t_{j}}{z}\right), z \in D^{-}
\end{array}\right.
$$


is a solution of the problem (2.11). The choice of the branches of corresponding multi-valued functions can be done as in the case $2 \mathrm{~b})$. Denoting $\omega(z):=$ $\prod_{j=1}^{n}\left(z-t_{j}\right)$ we construct the Lagrange polynomial with the knots $t_{j}$ for the function $g_{4}(t):=\frac{g_{2}(t)}{\left(t-t_{0}\right)^{\left.x_{0}-\mid x_{0}\right)}}$. Then the solution of the problem (2.5) in $\widetilde{\mathcal{A}}^{0,0}$ is given by the formulas (2.14) with the operator $\widetilde{\mathbf{S}}^{ \pm}$defined in (2.15), and the function $\widehat{g}(t):=g_{4}-\prod_{j=1}^{n} \frac{\omega(t) g_{4}\left(t_{j}\right)}{\omega /\left(t_{j}\right)\left(t-t_{j}\right)}$. The constants $C_{j}$ are chosen in the same way as in (2.16), and $C_{0}$ satisfying (2.17).

Remark 2.2. The properties of Cauchy type integral (see e.g. [1, p.61]) show us that

1) under the first (second) choice of $C_{j}$ in (2.16) the function $\Omega^{+}(t)\left(\Omega^{-}(t)\right.$ respectively) has zeros of at least an order $\lambda=\min \left\{\lambda_{G}, \lambda_{g}\right\}$ at $t_{j}$;

2 ) any point $t_{0}, t_{1}, \ldots, t_{n}$ is a zero for both functions $\Omega^{+}, \Omega^{-}$if the initial function $g$ vanishes at this point.

Remark 2.3. The choice (2.17) for $C_{0}$ is again possible because either for certain point $t \in L$ we have $\left(\widetilde{\mathbf{S}}^{+} \widehat{g}(t)\right) \in \widehat{\mathbb{C}} \backslash\left(\widetilde{\mathbf{S}}^{-} \widehat{g}\left(\overline{D^{-}}\right)\right)$, or $\left(\widetilde{\mathbf{S}}^{+} \widehat{g}(L)\right) \subset$ $\left(\widetilde{\mathbf{S}}^{-} \widehat{g}\left(\overline{D^{-}}\right)\right)$. The last is impossible because if so then there exist a curve $\widetilde{\Gamma}$ whose pre-image under the mapping $w^{-}:=\widetilde{\mathbf{S}}^{-} \widehat{g}$ and does not coincide with $L$. It means that there exist an analytic function which maps disconnected domain onto the whole complex plane.

ii) The second approach deals with idea of $2 \mathrm{~b}$ ), namely, we fix a number $r \in(0,1)$ such that $r \leq \min \left\{\lambda_{G}, \lambda_{g}, 1-\epsilon\right\}, r \cdot n=\chi_{0}$ for certain $n \in \mathbb{N}$. Having chosen $n$ points $t_{j}, j=1, \ldots, n$, on $L$ we introduce the solution of (2.11) $X^{ \pm}$by the formulas:

$$
\left\{\begin{aligned}
X^{+}(z) & :=\prod_{j=1}^{n}\left(z-t_{j}\right)^{r}, z \in D^{+} \\
X^{+}(z) & :=\prod_{j=1}^{n}\left(1-\frac{t_{j}}{z}\right)^{r}, z \in D^{-}
\end{aligned}\right.
$$

with the appropriate choice of the branches of multi-valued functions (see (2.20). Then the solution of the problem (2.5) in the class $\widetilde{\mathcal{A}}^{0,0}$ is delivered in (2.21) with the choice of the constant $C_{0}$ according to (2.22).

Remark 2.4. The difference between two approaches is in the following: the first construction prescribe either to $\Omega^{+}$, or to $\Omega^{-}$to be vanishing at the point $t_{j}$ (and even minimal order of zeros at these points), but the second one guarantees only that the solution cannot be vanishing at no other point despite (possibly) $t_{j}$. 
It leads to two different meaning of the solution to the problem (2.1) in $\widetilde{\mathcal{A}}^{k, l}$. One can search or for solution which ought to have boundary zeros of prescribed minimal order, or for solution which possibly have zeros at certain points on the boundary.

b) $\chi_{0} \in \mathbb{R}_{-} \backslash \mathbb{Z}$, i.e. $\chi_{0}$ is a negative noninteger number. It should be noted as in the case $2 b$ ), that taking solution of factorization problem in the form (2.18) one get very strong solvability condition on the initial data. It means that in principle it is difficult to get the solution of (2.1) of the first type described in the previous Remark.

Therefore we look only for the solution of the second type. Let us fix a number $r \in(0,1)$ such that $r \leq \min \left\{\lambda_{G}, \lambda_{g} 1-\epsilon\right\}, r \cdot n=-\chi_{0}$ for certain $n \in \mathbb{N}$. Having chosen $n$ (different) points $t_{1}, \ldots, t_{n} \in L$ we take the solution of (2.11) in the form (2.19) with the choice of branches as in (2.20). Then the formulas (2.21) give us solution of the problem (2.5) in the class $\widetilde{\mathcal{A}}^{0,0}$ if and only if the solvability conditions (2.23) do satisfy.

Proposition 2.3. Let $(k, l) \in \mathbb{N}_{0} \times \mathbb{N}_{0}$ be such that $\chi_{0} \neq 0$ is an irrational number.

If $\chi_{0} \in \mathbb{R}_{+} \backslash \mathbb{Z}$ then the unique (up to the constant $C_{0}$ ) solution of (2.1) in $\widetilde{\mathcal{A}}^{k, l}$ can be represented in the form (2.10) with $\Omega^{ \pm}$either given by the formulas (2.14) with $X^{ \pm}$in (2.24), $C_{j}$ in (2.16), and $C_{0}$ satisfying (2.17), or $\Omega^{ \pm}$given by the formulas (2.21) with $X^{ \pm}$in (2.25), and $C_{0}$ satisfying (2.22).

The solution of the first type necessarily has zeros at the points $t_{j}$ of the order not less than $\lambda$, but the second one is nonvanishing on L inspite possibly the points $t_{j}$.

If $\chi_{0} \in \mathbb{R}_{-} \backslash \mathbb{Z}$ then the solution of the problem (2.1) can be also represented in the form (2.10) with $\Omega^{ \pm}$given by the formulas (2.21) with $X^{ \pm}$in (2.19), $C_{j}$ in (2.16). This solution belongs to $\widetilde{\mathcal{A}}^{k, l}$ if the solvability conditions (2.23) do satisfy.

\section{SOLVABILITY THEOREM}

Combining the above results we get the following

Theorem 3.1. Let the winding number $\chi$ of the coefficient $G$ of the nonlinear conjugation problem (2.1) is nonnegative integer number, i.e. $\chi \geq 0$. Then:

i) the problem (2.1) has a solution (2.10) with $\Omega^{ \pm}$in (2.4) and with $C_{0}$ chosen in accordance with (2.8) (respectively (2.8)) only in those classes $\mathcal{A}^{k, l}$ (resp. in $\widetilde{\mathcal{A}}^{k, l}$ ) for which the condition (2.6) holds;

ii) the problem (2.1) has a solution (2.10) of the first type, i.e. with $\Omega^{ \pm}$ in (2.14), and with $C_{0}$ chosen in accordance with (2.17) only in those classes $\widetilde{\mathcal{A}}^{k, l}$ for which $\chi_{0}:=\chi-k \alpha-l \beta$ is positive; 
iii) the problem (2.1) has a solution (2.10) of the second type, i.e. with $\Omega^{ \pm}$ in (2.21), $X^{ \pm}$given in (2.19), in those classes $\widetilde{\mathcal{A}}^{k, l}$ for which $\chi_{0}$ is negative number if the solvability conditions (2.23) do satisfy;

If $\chi<0$ then:

i) the problem (2.1) has no solution in the class $\mathcal{A}^{k, l}$;

ii) the problem (2.1) has a solution (2.10) of the second type, i.e. with $\Omega^{ \pm}$ in (2.21), $X^{ \pm}$given in (2.19), in those classes $\widetilde{\mathcal{A}}^{k, l}$ for which $\chi_{0}$ is negative if the solvability conditions (2.23) do satisfy.

Aknowledgement The work is partially supported by Belarusian Fund for Fundamental Scientific Research. The author expresses his deep gratitude to Prof. S. Rutkauskas for the warm hospitality during the Conference MMA99.

\section{REFERENCES}

[1] F.D. Gakhov. Boundary Value Problems. Nauka, Moscow, (3rd English transl. of the 2nd ed. by Pergamon Press, Oxford. 1977 (in Russian); 1966 (in English)

[2] N.I. Muskhelishvili. Singular Integral Equations. Nauka, Moscow, (3rd ed.); Engl. transl. of the 1st ed. by Noordhoff, Groninge, 1968. (in Russian); 1953 (in English)

[3] V.K. Natalevich. Nonlinear singular integral equations and nonlinear boundary value problems of the theory of analytic functions. Uchen. zap. Kazan Univ., 112 (10), 1952, 155 -190.(Russian)

[4] Yu.V. Obnosov. Some nonlinear boundary value problems solving in closed form. In: Nauchnye Trudy Jubilejnogo Seminara po Kraevym Zadacham, Universities Press, Minsk, (Ed.) V.P. Platonov, 1985, 85 - 96.(in Russian)

[5] L.von Wolfersdorf. On the theory of the nonlinear Hilbert problem for holomorphic functions. Partial Differential Equations with Complex Analysis (H. Begehr, A. Jeffrey eds.), Addison Wesley Longman, Harlow, 1992, 134 - 149.

[6] A.I. Guseinov and Kh. ShMukhtarov. Introduction to the Theory of Nonlinear Singular Integral Equations. Nauka, Moscow, 1980. (in Russian)

[7] V.V. Mityushev and S.V. Rogosin. Constructive Methods for Linear and Nonlinear Boundary Value Problems for Analytic Functions. Theory and Applications, CRC Press, Boca Raton, Fl. 1999 (to appear)

[8] N.V. Govorov and N.K. Kuznetsov. On nonlinear conjugation boundary value problem for disconnected contour. In: Theory of Functions, Functional Analysis and Applications, Khar'kov, (20), 1974, 49 - 63.(in Russian)

[9] F.D. Gakhov. On nonlinear boundary value problem generalizing the Riemann boundary value problem. Doklady AN SSSR , 181 (2), 1968, 271 - 274 .(in Russian)

[10] F.D. Gakhov. On nonlinear boundary value problem with addmissible zeroes on the contour. Doklady AN SSSR, 210 (6), 1973, 21 - 24.(in Russian)

[11] N.A. Rysjuk. On nonlinear boundary value problem of the Riemann type. Doklady AN bSSR, 21 (4), 1977, 51 - 56 .(in Russian) 


\section{ANALIZINIŲ FUNKCIJŲ NETIESINIO SUJUNGIMO KRAS̆TINIO UŽDAVINIO KOREKTIŠKUMAS}

S.V. ROGOSIN

Nagrinëjamas analizinių funkcijų netiesinio sujungimo kraštinis uždavinys. Analizuojami laipsninio tipo netiesiškumai. Pagrindinis darbo tikslas yra korektiškai suformuluoti uždavini, t.y. rasti tokiu funkciju klasę, kurioje šis uždavinys turi vienintelį sprendini. Parodyta, kad tokią funkcijų klasę sudaro funkcijos su iš anksto užduota nulių aibe srityje ir /arba ant jos kontūro. 